PROCEEDINGS OF THE

AMERICAN MATHEMATICAL SOCIETY

Volume 130, Number 8, Pages 2403-2412

S 0002-9939(02)06329-3

Article electronically published on January 23, 2002

\title{
ON A LOCAL VERSION OF THE ALEKSANDROV-FENCHEL INEQUALITY FOR THE QUERMASSINTEGRALS OF A CONVEX BODY
}

\author{
A. GIANNOPOULOS, M. HARTZOULAKI, AND G. PAOURIS
}

(Communicated by N. Tomczak-Jaegermann)

\begin{abstract}
We discuss the analogue in the Brunn-Minkowski theory of the inequalities of Marcus-Lopes and Bergstrom about symmetric functions of positive reals and determinants of symmetric positive matrices respectively. We obtain a local version of the Aleksandrov-Fenchel inequality $W_{i}^{2} \geq W_{i-1} W_{i+1}$ which relates the quermassintegrals of a convex body $K$ to those of an arbitrary hyperplane projection of $K$. A consequence is the following fact: for any convex body $K$, for any $(n-1)$-dimensional subspace $E$ of $\mathbb{R}^{n}$ and any $t>0$,

$$
\frac{\left|P_{E}(K)+t D_{E}\right|}{\left|P_{E}(K)\right|} \leq \frac{\left|K+2 t D_{n}\right|}{|K|}
$$
\end{abstract}

where $D$ denotes the Euclidean unit ball and $|\cdot|$ denotes volume in the appropriate dimension.

\section{INTRODUCTION}

The starting point of this paper is an inequality of Marcus and Lopes [9] about symmetric functions of positive real numbers. The $i$-th elementary symmetric function $E_{i}(x)$ of an $n$-tuple $x=\left(x_{1}, \ldots, x_{n}\right)$ of positive reals is defined by $E_{0}(x)=1$ and

$$
E_{i}(x)=\sum_{1 \leq j_{1}<\cdots<j_{i} \leq n} x_{j_{1}} x_{j_{2}} \cdots x_{j_{i}}, \quad 1 \leq i \leq n .
$$

In particular, $E_{1}(x)=\sum_{i=1}^{n} x_{i}$ and $E_{n}\left(x_{1}, \ldots, x_{n}\right)=\prod_{i=1}^{n} x_{i}$. With this notation, for every $i=1, \ldots, n$ and all positive $n$-tuples $x, y$ one has

$$
\frac{E_{i}(x+y)}{E_{i-1}(x+y)} \geq \frac{E_{i}(x)}{E_{i-1}(x)}+\frac{E_{i}(y)}{E_{i-1}(y)}
$$

A formal consequence of the arithmetic-geometric means inequality and (1.1) is the inequality

$$
\left[E_{i}(x+y)\right]^{1 / i} \geq\left[E_{i}(x)\right]^{1 / i}+\left[E_{i}(y)\right]^{1 / i}
$$

(see [3], $\S 1.33$ and $\S 1.34$, for proofs and extensions of these facts).

Received by the editors December 20, 2000 and, in revised form, March 16, 2001.

1991 Mathematics Subject Classification. Primary 52A20; Secondary 52A39, 52A40.

Key words and phrases. Mixed volumes, Aleksandrov-Fenchel inequality. 
An inequality of Bergstrom [1], which is the matrix analogue of (1.1) (see also [3], $\S 2.17$ ), states that if $A$ and $B$ are symmetric positive definite matrices and $A_{i}, B_{i}$ denote the submatrices obtained by deleting the $i$-th row and column, then

$$
\frac{\operatorname{det}(A+B)}{\operatorname{det}\left(A_{i}+B_{i}\right)} \geq \frac{\operatorname{det}(A)}{\operatorname{det}\left(A_{i}\right)}+\frac{\operatorname{det}(B)}{\operatorname{det}\left(B_{i}\right)}
$$

This is generalized by Ky Fan [7] in the form

$$
\left(\frac{\operatorname{det}(A+B)}{\operatorname{det}\left(A_{k}+B_{k}\right)}\right)^{1 / k} \geq\left(\frac{\operatorname{det}(A)}{\operatorname{det}\left(A_{k}\right)}\right)^{1 / k}+\left(\frac{\operatorname{det}(B)}{\operatorname{det}\left(B_{k}\right)}\right)^{1 / k}
$$

where $A_{k}$ is the submatrix of $A$ obtained by deleting $k$ rows and the corresponding columns of $A$. When $k=n$, this reduces to Minkowski's inequality $[\operatorname{det}(A+B)]^{1 / n} \geq$ $[\operatorname{det} A]^{1 / n}+[\operatorname{det} B]^{1 / n}$.

There is a remarkable similarity between inequalities about symmetric functions (or determinants of symmetric matrices) and inequalities about the mixed volumes of convex bodies. In particular, the analogue of (1.2) or (1.4) in the Brunn-Minkowski theory is

$$
W_{i}(A+B)^{1 /(n-i)} \geq W_{i}(A)^{1 /(n-i)}+W_{i}(B)^{1 /(n-i)}, \quad i=0, \ldots, n-1,
$$

which holds true for any pair of convex bodies $A$ and $B$ in $\mathbb{R}^{n}$. This is a consequence of the Aleksandrov-Fenchel inequality (see the next section for the definition of the quermassintegrals $W_{i}(\cdot)$ and background material).

In view of these analogies, V. Milman asked if there is a version of (1.1) or (1.3) in the theory of mixed volumes. As we shall see in the next section, one can prove a similar statement when $B$ is a ball:

Theorem 1.1. Let $A$ be a convex body and $B$ be a ball in $\mathbb{R}^{n}$. Then

$$
\frac{W_{i}(A+B)}{W_{i+1}(A+B)} \geq \frac{W_{i}(A)}{W_{i+1}(A)}+\frac{W_{i}(B)}{W_{i+1}(B)}
$$

for all $i \in\{0, \ldots, n-1\}$.

The referee of this paper informed us that the case $i=0$ of this theorem has already appeared in the literature; in [5] it is stated as an open question whether this inequality holds for an arbitrary pair of nonempty compact convex sets $A$ and $B$. It is also mentioned that (1.6) follows directly from the Aleksandrov-Fenchel inequality when $i=0$ and $B$ is a ball.

The answer to the above question is negative; it can be proved that (1.6) is true in full generality only when $i=n-2$ or $i=n-1$ (the details will appear in [6]). However, it is an interesting question to describe the class $\mathcal{B}$ of compact convex subsets $B$ of $\mathbb{R}^{n}$ for which the weaker inequality

$$
\frac{W_{i}(A+B)}{W_{i+1}(A+B)} \geq \frac{W_{i}(A)}{W_{i+1}(A)}
$$

holds true for every convex body $A$. In particular, if line segments belong to this class, then taking $i=0, A=K$, and $B=[-\theta, \theta]$ for any $\theta \in S^{n-1}$ we see that

$$
\frac{\partial\left(P_{\theta^{\perp}}(K)\right)}{\left|P_{\theta^{\perp}}(K)\right|} \leq \frac{\partial(K)}{|K|}
$$

for every convex body $K$ in $\mathbb{R}^{n}$, where $P_{\theta^{\perp}}$ denotes orthogonal projection onto $\theta^{\perp}$ and $\partial(\cdot)$ is surface area in the appropriate dimension. This corresponds to 
the following isoperimetric problem (which we conjecture to have an affirmative answer):

Question 1. Let $\mathcal{A}$ denote the class of all convex bodies whose projection onto $E$ is a given convex body $A$ (this is the canal class of $A$ in the terminology of [10]). Is it true that the infimum of the ratio $\partial(K) /|K|$ over all $K \in \mathcal{A}$ is "attained" for a cylinder of "infinite length" in $\mathcal{A}$ ?

We present two approaches to this question. The first one is based on a local version of the Aleksandrov-Fenchel inequalities for the quermassintegrals of a convex body. By this we mean a set of inequalities involving the quermassintegrals of the body and of an arbitrary hyperplane projection, which imply the classical inequalities $W_{i}(K)^{2} \geq W_{i+1}(K) W_{i-1}(K)$ up to constant by integration on the appropriate Grassmannian. The precise statement is the following.

Theorem 1.2. Let $K$ be a convex body in $\mathbb{R}^{n}$ and let $E$ be an $(n-1)$-dimensional subspace of $\mathbb{R}^{n}$. Then,

$$
\frac{W_{i+1}(K)}{2 W_{i}(K)} \leq \frac{W_{i}^{\prime}\left(P_{E}(K)\right)}{W_{i-1}^{\prime}\left(P_{E}(K)\right)} \leq \frac{2 W_{i}(K)}{W_{i-1}(K)}
$$

for every $i \in\{1, \ldots, n-1\}$.

Here, the prime in $W_{i}^{\prime}$ means that the quermassintegrals of $P_{E}(K)$ are being considered in the appropriate dimension $n-1$. The most interesting case is when $i=1$. Then, Theorem 1.2 implies that

$$
\frac{\partial\left(P_{E}(K)\right)}{\left|P_{E}(K)\right|} \leq \frac{2(n-1)}{n} \frac{\partial(K)}{|K|}
$$

for every $(n-1)$-dimensional subspace $E$. The question is whether this inequality holds true with constant 1 instead of $2(n-1) / n$.

Our second approach, which is more elementary, is based on a local version of the Loomis-Whitney inequality about coordinate projections of a convex body.

The results of this paper are also related to the following question:

Question 2. Let $K$ be a convex body in $\mathbb{R}^{n}$. For every $t>0$, consider the $t$ extension $K_{t}:=K+t D_{n}$ of $K$, where $D_{n}$ is the Euclidean unit ball. Is it true that the ratio $\left|K_{t}\right| /|K|$ decreases under projection onto any subspace $E$ of $\mathbb{R}^{n}$ ?

It is clear by induction that a positive answer to this question in the case $\operatorname{dim}(E)=n-1$ is enough for the general case. What we are able to prove is the following fact.

Theorem 1.3. Let $K$ be a convex body in $\mathbb{R}^{n}$ and let $E$ be an $(n-1)$-dimensional subspace of $\mathbb{R}^{n}$. Then,

$$
\frac{\left|P_{E}(K)+t D_{E}\right|}{\left|P_{E}(K)\right|} \leq \frac{\left|K+2 t D_{n}\right|}{|K|} .
$$

The paper is organized as follows. In Section 2 we recall definitions and basic facts from the classical Brunn-Minkowski theory (for a detailed exposition we refer the reader to the books [4] and [10]). We also prove Theorem 1.1. In Section 3 we prove Theorems 1.2 and 1.3; our approach makes use of the general AleksandrovFenchel inequality. In Section 4 we prove a local Loomis-Whitney type inequality which leads to (1.10). 


\section{NOTATION AND BACKGROUND MATERIAL}

We shall work in $\mathbb{R}^{n}$, which is equipped with an inner product $\langle\cdot, \cdot\rangle$ and a fixed orthonormal basis $\left\{e_{1}, \ldots, e_{n}\right\}$. We denote by $D_{n}$ and $S^{n-1}$ the unit ball and the unit sphere in $\mathbb{R}^{n}$ respectively. We write $\sigma$ for the normalized rotationally invariant probability measure on $S^{n-1}$ and $\mu$ for the Haar probability measure on the orthogonal group $O(n)$. Let $G_{n, k}$ denote the Grassmannian of all $k$-dimensional subspaces of $\mathbb{R}^{n}$. Then, $O(n)$ equips $G_{n, k}$ with a Haar probability measure $\nu_{n, k}$.

Let $\mathcal{K}_{n}$ denote the class of all nonempty compact convex subsets of $\mathbb{R}^{n}$. If $K \in \mathcal{K}_{n}$ has nonempty interior, we will say that $K$ is a convex body. If $A \in \mathcal{K}_{n}$, we will denote by $|A|$ the volume of $A$ in the appropriate affine subspace. The volume of $D_{n}$ is $\omega_{n}$.

If $\theta \in S^{n-1}$, then $\theta^{\perp}=\left\{x \in \mathbb{R}^{n}:\langle x, \theta\rangle=0\right\}$. The section of $K \in \mathcal{K}_{n}$ with a subspace $E$ of $\mathbb{R}^{n}$ is $K \cap E$, and the orthogonal projection of $K$ onto $E$ is denoted by $P_{E}(K)$.

Minkowski's theorem provides a fundamental relation between volume and the operations of addition and multiplication of convex bodies by nonnegative reals: If $K_{1}, \ldots, K_{m} \in \mathcal{K}_{n}, m \in \mathbb{N}$, then the volume of $t_{1} K_{1}+\cdots+t_{m} K_{m}$ is a homogeneous polynomial of degree $n$ in $t_{i} \geq 0$ (see [4], 10]). That is,

$$
\left|t_{1} K_{1}+\cdots+t_{m} K_{m}\right|=\sum_{1 \leq i_{1}, \ldots, i_{n} \leq m} V\left(K_{i_{1}}, \ldots, K_{i_{n}}\right) t_{i_{1}} \cdots t_{i_{n}},
$$

where the coefficients $V\left(K_{i_{1}}, \ldots, K_{i_{n}}\right)$ are chosen to be invariant under permutations of their arguments. The coefficient $V\left(K_{i_{1}}, \ldots, K_{i_{n}}\right)$ is called the mixed volume of the $n$-tuple $\left(K_{i_{1}}, \ldots, K_{i_{n}}\right)$.

Steiner's formula is a special case of Minkowski's theorem; the volume of $K+t D_{n}$, $t>0$, can be expanded as a polynomial in $t$ :

$$
\left|K+t D_{n}\right|=\sum_{i=0}^{n}\left(\begin{array}{c}
n \\
i
\end{array}\right) W_{i}(K) t^{i}
$$

where $W_{i}(K):=V\left(K, n-i ; D_{n}, i\right)$ is the $i$-th quermassintegral of $K$.

The Aleksandrov-Fenchel inequality states that if $K, L, K_{3}, \ldots, K_{n} \in \mathcal{K}_{n}$, then

$$
V\left(K, L, K_{3}, \ldots, K_{n}\right)^{2} \geq V\left(K, K, K_{3}, \ldots, K_{n}\right) V\left(L, L, K_{3}, \ldots, K_{n}\right) .
$$

In particular, this implies that the sequence $\left(W_{0}(K), \ldots, W_{n}(K)\right)$ is log-concave. In other words,

$$
W_{j}^{k-i} \geq W_{i}^{k-j} W_{k}^{j-i}
$$

if $0 \leq i<j<k \leq n$. From the Aleksandrov-Fenchel inequality one can also recover the Brunn-Minkowski inequality as well as the following generalization for the quermassintegrals:

$$
W_{i}(K+L)^{1 /(n-i)} \geq W_{i}(K)^{1 /(n-i)}+W_{i}(L)^{1 /(n-i)}, \quad i=0, \ldots, n-1 .
$$

Let $\partial(K)$ denote the surface area of $K$. From Steiner's formula and the definition of surface area we see that $\partial(K)=n W_{1}(K)$.

Let us finally mention Kubota's integral formula

$$
W_{i}(K)=\frac{\omega_{n}}{\omega_{n-i}} \int_{G_{n, n-i}}\left|P_{E}(K)\right| \nu_{n, n-i}(d E), \quad 1 \leq i \leq n-1 .
$$


The case $i=1$ is Cauchy's surface area formula

$$
\partial(K)=\frac{\omega_{n}}{n \omega_{n-1}} \int_{S^{n-1}}\left|P_{u^{\perp}}(K)\right| \sigma(d u) .
$$

Using (2.4) one can easily prove Theorem 1.1.

Theorem. Let $A$ be any convex body and let $B$ be a ball in $\mathbb{R}^{n}$. Then,

$$
\frac{W_{i}(A+B)}{W_{i+1}(A+B)} \geq \frac{W_{i}(A)}{W_{i+1}(A)}+\frac{W_{i}(B)}{W_{i+1}(B)}
$$

for all $i \in\{0, \ldots, n-1\}$.

Proof. We may assume that $B=t D_{n}$ for some $t>0$. For every $i \in\{0, \ldots, n-1\}$ we define $f_{i}(s)=W_{i}\left(A+s D_{n}\right)$. Then, by the linearity of the mixed volumes we see that

$$
f_{i}(s+\varepsilon)=f_{i}(s)+\varepsilon(n-i) f_{i+1}(s)+O\left(\varepsilon^{2}\right)
$$

for all $i \leq n-1$, and therefore

$$
f_{i}^{\prime}(s)=(n-i) f_{i+1}(s) .
$$

From the Aleksandrov-Fenchel inequalities (2.4) we have $f_{i+1}^{2}(s) \geq f_{i}(s) f_{i+2}(s)$ for all $i \leq n-2$. It follows that

$$
\begin{aligned}
f_{i}^{\prime}(s) f_{i+1}(s)-f_{i}(s) f_{i+1}^{\prime}(s) & =(n-i) f_{i+1}^{2}(s)-(n-i-1) f_{i}(s) f_{i+2}(s) \\
& =f_{i+1}^{2}(s)+(n-i-1)\left[f_{i+1}^{2}(s)-f_{i}(s) f_{i+2}(s)\right] \\
& \geq f_{i+1}^{2}(s)
\end{aligned}
$$

if $0 \leq i \leq n-2$. This implies that the function $g_{i}: \mathbb{R}^{+} \rightarrow \mathbb{R}^{+}$defined by $g_{i}(s)=W_{i}\left(A+s D_{n}\right) / W_{i+1}\left(A+s D_{n}\right)$ satisfies $g_{i}^{\prime}(s) \geq 1$. Hence, $g_{i}(t) \geq g_{i}(0)+t$ for every $t \geq 0$. This is exactly the statement of the theorem.

Observe that when $i=n-1$, the theorem reduces to the inequality

$$
W_{n-1}(A+B) \geq W_{n-1}(A)+W_{n-1}(B),
$$

which holds as an equality for every pair of convex bodies; mean width is linear with respect to Minkowski addition. This completes the proof.

An immediate consequence of the proof of Theorem 1.1 is the following fact:

Corollary 2.1. Let $K$ be a convex body in $\mathbb{R}^{n}$. For every $0 \leq j<i \leq n-1$, the function

$$
\frac{W_{j}\left(K+t D_{n}\right)}{W_{i}\left(K+t D_{n}\right)}
$$

is an increasing function of $t \in[0,+\infty)$.

Let $k \in\{1, \ldots, n-1\}$. From Corollary 2.1, we readily see that the function $W_{k}\left(K+t D_{n}\right) / W_{0}\left(K+t D_{n}\right)$ is increasing. Kubota's formula (2.6) shows that

$$
\int_{G_{n, n-k}}\left|P_{E}(K)\right| \nu_{n, n-k}(d E) \geq \frac{|K|}{\left|K+t D_{n}\right|} \int_{G_{n, n-k}}\left|P_{E}(K)+t D_{E}\right| \nu_{n, n-k}(d E)
$$


for every $t>0$, hence our question about $t$-extensions has an affirmative answer (for any codimension $k$ ) on the average. In particular, for every $t>0$ we have

$$
\frac{\left|K+t D_{n}\right|}{\partial\left(K+t D_{n}\right)} \geq \frac{|K|}{\partial(K)}
$$

\section{Local version of the Aleksandrov-Fenchel inequality}

If $u \in S^{n-1}$, we write $J_{u}$ for the line segment $[o, u]$. Computing the volume of $K+t J_{u}$, we see that $n V\left(K, n-1 ; J_{u}\right)=\left|P_{E}(K)\right|$, where $E=u^{\perp}$. Linearity of mixed volumes shows that

$$
n V\left(K_{1}, \ldots, K_{n-1}, J_{u}\right)=V_{E}\left(P_{E}\left(K_{1}\right), \ldots, P_{E}\left(K_{n-1}\right)\right)
$$

for all $K_{1}, \ldots, K_{n-1} \in \mathcal{K}_{n}$. In fact, there is a generalization of (3.1), which is due to Fedotov (see [4]):

Lemma 3.1. Let $E \in G_{n, k}$ and let $L_{1}, \ldots, L_{n-k}$ be compact convex subsets of $E^{\perp}$. If $K_{1}, \ldots, K_{k} \in \mathcal{K}_{n}$, then

$$
\left(\begin{array}{l}
n \\
k
\end{array}\right) V\left(K_{1}, \ldots, K_{k}, L_{1}, \ldots, L_{n-k}\right)=V_{E}\left(P_{E}\left(K_{1}\right), \ldots, P_{E}\left(K_{k}\right)\right) V_{E^{\perp}}\left(L_{1}, \ldots, L_{n-k}\right),
$$

where $V_{E}, V_{E^{\perp}}$ denote mixed volumes on $E, E^{\perp}$ respectively.

We will also need a consequence of the Aleksandrov-Fenchel inequality (for historical remarks on inequalities of this type see [10], Section 6.4).

Lemma 3.2. Let $\mathcal{C}=\left(K_{3}, \ldots, K_{n}\right)$, where $K_{j} \in \mathcal{K}_{n}, j=3, \ldots, n$. If $A, B \in \mathcal{K}_{n}$, we denote $V(A, B, \mathcal{C})$ by $V(A, B)$. Then, for all $A, B, C \in \mathcal{K}_{n}$ we have either $V(B, C) V(A, A) \geq V(B, A) V(C, A)$ or

$$
\begin{aligned}
(V(A, B) V(A, C)-V(B, C) V(A, A))^{2} \leq & {\left[V(A, B)^{2}-V(A, A) V(B, B)\right] } \\
& \cdot\left[V(A, C)^{2}-V(A, A) V(C, C)\right]
\end{aligned}
$$

Proof. By the Aleksandrov-Fenchel inequality, for all $t, s \geq 0$ we have

$$
V(B+t A, C+s A)^{2}-V(B+t A, B+t A) V(C+s A, C+s A) \geq 0,
$$

and, using the linearity of mixed volumes, we arrive at

$$
\begin{aligned}
0 \leq & g(t, s)+t^{2}\left(V(C, A)^{2}-V(A, A) V(C, C)\right) \\
& +s^{2}\left(V(B, A)^{2}-V(A, A) V(B, B)\right) \\
& +2 t s(V(B, C) V(A, A)-V(B, A) V(C, A)),
\end{aligned}
$$

where $g$ is a linear function of $t$ and $s$. It follows that the quadratic term is nonnegative and hence, either $V(B, C) V(A, A) \geq V(B, A) V(C, A)$ or its discriminant is non-positive. This proves the lemma.

Let $K$ be a convex body in $\mathbb{R}^{n}$. For every $i \in\{1, \ldots, n-1\}$, we define the parameter

$$
d_{i}:=d_{i}(K)=\frac{W_{i-1}(K) W_{i+1}(K)}{W_{i}^{2}(K)} .
$$

It is clear that $d_{i}(K)>0$, and the Aleksandrov-Fenchel inequalities show that $d_{i}(K) \leq 1$. In the symmetric case, Schneider [11] has proved that $d_{i}(K)=1$ if and only if $K$ is an $(n-i-1)$-tangential body of a ball. 
Theorem 3.3. Let $K$ be a convex body in $\mathbb{R}^{n}$. For every $(n-1)$-dimensional subspace $E$ of $\mathbb{R}^{n}$ and every $i \in\{1, \ldots, n-2\}$, we have

$$
\left(1-\sqrt{1-d_{i}}\right) \frac{W_{i}(K)}{W_{i-1}(K)} \leq \frac{W_{i}^{\prime}\left(P_{E}(K)\right)}{W_{i-1}^{\prime}\left(P_{E}(K)\right)} \leq\left(1+\sqrt{1-d_{i}}\right) \frac{W_{i}(K)}{W_{i-1}(K)} .
$$

Notation. We write $W_{i}^{\prime}\left(P_{E}(K)\right)$ for $V_{E}\left(P_{E}(K), n-1-i ; D_{E}, i\right)$, where $V_{E}$ denotes mixed volume in $E$.

Proof. Let $\mathcal{C}$ be the $(n-2)$-tuple $\left(K, n-i-1 ; D_{n}, i-1\right)$ and assume that $E=u^{\perp}$, $u \in S^{n-1}$.

We first set $A=K, B=J_{u}$ and $C=D_{n}$. Observe that $V\left(J_{u}, J_{u}\right)=0$. By Lemma 3.2, there are two possibilities: either

$$
V\left(J_{u}, D_{n}\right) V(K, K) \geq V\left(J_{u}, K\right) V\left(K, D_{n}\right)
$$

which, by Lemma 3.1, implies

$$
\frac{W_{i}(K)}{W_{i-1}(K)} \leq \frac{W_{i}^{\prime}\left(P_{E}(K)\right)}{W_{i-1}^{\prime}\left(P_{E}(K)\right)}
$$

or

$$
\left.\frac{W_{i}(K)}{W_{i-1}(K)}-\frac{V\left(J_{u}, D_{n}\right)}{V\left(J_{u}, K\right)} \leq \frac{1}{W_{i-1}(K)}\left(W_{i}(K)^{2}-W_{i-1}(K) W_{i+1}(K)\right)\right)^{1 / 2}
$$

and Lemma 3.1 gives

$$
\left(1-\sqrt{1-d_{i}}\right) \frac{W_{i}(K)}{W_{i-1}(K)} \leq \frac{V\left(J_{u}, D_{n}\right)}{V\left(J_{u}, K\right)}=\frac{W_{i}^{\prime}\left(P_{E}(K)\right)}{W_{i-1}^{\prime}\left(P_{E}(K)\right)} .
$$

From (3.2) and (3.4) we get the left-hand side inequality of the theorem. For the right-hand side inequality, we choose $A=D_{n}, B=J_{u}$ and $C=K$ in Lemma 3.2, and follow the same steps. We either have

$$
\frac{W_{i}^{\prime}\left(P_{E}(K)\right)}{W_{i-1}^{\prime}\left(P_{E}(K)\right)} \leq \frac{W_{i+1}(K)}{W_{i}(K)} \leq \frac{W_{i}(K)}{W_{i-1}(K)}
$$

or

$$
\frac{V\left(J_{u}, D_{n}\right)}{V\left(J_{u}, K\right)}-\frac{W_{i+1}(K)}{W_{i}(K)} \leq \frac{V\left(J_{u}, D_{n}\right)}{V\left(J_{u}, K\right)}\left(W_{i}(K)^{2}-W_{i-1}(K) W_{i+1}(K)\right)^{1 / 2},
$$

which implies

$$
\frac{W_{i}^{\prime}\left(P_{E}(K)\right)}{W_{i-1}^{\prime}\left(P_{E}(K)\right)} \leq \frac{1}{1-\sqrt{1-d_{i}}} \frac{W_{i+1}(K)}{W_{i}(K)}=\left(1+\sqrt{1-d_{i}}\right) \frac{W_{i}(K)}{W_{i-1}(K)} .
$$

This completes the proof.

Proof of Theorems 1.2 and 1.3. Since $d_{i}(K) \leq 1$ for all $1 \leq i \leq n-1$, simple algebra gives

$$
\frac{W_{i+1}(K)}{2 W_{i}(K)} \leq \frac{W_{i}^{\prime}\left(P_{E}(K)\right)}{W_{i-1}^{\prime}\left(P_{E}(K)\right)} \leq \frac{2 W_{i}(K)}{W_{i-1}(K)}
$$

for every $(n-1)$-dimensional subspace $E$ of $\mathbb{R}^{n}$ and every $i \in\{1, \ldots, n-1\}$. This proves Theorem 1.2. 
For the proof of Theorem 1.3, we first apply successively (3.8) to obtain

$$
\frac{W_{i}^{\prime}\left(P_{E}(K)\right)}{\left|P_{E}(K)\right|} \leq \frac{2^{i} W_{i}(K)}{|K|}
$$

for all $i \in\{1, \ldots, n-1\}$, and then use Steiner's formula:

$$
\begin{aligned}
\left|P_{E}(K)+t D_{E}\right|-\left|P_{E}(K)\right| & =\sum_{i=1}^{n-1}\left(\begin{array}{c}
n-1 \\
i
\end{array}\right) W_{i}^{\prime}\left(P_{E}(K)\right) t^{i} \\
& \leq \frac{\left|P_{E}(K)\right|}{|K|} \sum_{i=1}^{n-1} \frac{n-i}{n}\left(\begin{array}{c}
n \\
i
\end{array}\right) W_{i}(K)(2 t)^{i},
\end{aligned}
$$

which implies

$$
\frac{\left|P_{E}(K)+t D_{E}\right|-\left|P_{E}(K)\right|}{\left|P_{E}(K)\right|} \leq \frac{n-1}{n} \frac{\left|K+2 t D_{n}\right|-|K|}{|K|} .
$$

This is slightly stronger than the assertion of Theorem 1.3.

\section{A local Loomis-Whitney Inequality}

Let $K$ be a convex body in $\mathbb{R}^{n}, n \geq 2$. We write $P_{i}(K)$ for the orthogonal projection of $K$ onto $e_{i}^{\perp}, i=1, \ldots, n$. The Loomis-Whitney inequality [8] compares $|K|$ with the geometric mean of $\left|P_{i}(K)\right|$ :

$$
|K|^{n-1} \leq \prod_{i=1}^{n}\left|P_{i}(K)\right|
$$

We will show that one can estimate $|K|$ using a smaller number of projections.

Lemma 4.1. Let $K$ be a convex body in $\mathbb{R}^{n}$ and let $i \neq j \in\{1, \ldots, n\}$. If $P_{i j}(K)=$ $P_{\left\langle e_{i}, e_{j}\right\rangle}(K)$, then

$$
\left|P_{i}(K)\right| \cdot\left|P_{j}(K)\right| \geq \frac{n}{2(n-1)}|K| \cdot\left|P_{i j}(K)\right| .
$$

For the proof of Lemma 4.1, we will use a classical inequality of Berwald [2].

Lemma 4.2. Let $A$ be a convex body in $\mathbb{R}^{k}$, and let $\phi: A \rightarrow \mathbb{R}^{+}$be a concave function. Then, for every $0<p<q$,

$$
\left[\left(\begin{array}{c}
k+q \\
k
\end{array}\right) \frac{1}{|A|} \int_{A}|\phi(x)|^{q} d x\right]^{1 / q} \leq\left[\left(\begin{array}{c}
k+p \\
k
\end{array}\right) \frac{1}{|A|} \int_{A}|\phi(x)|^{p} d x\right]^{1 / p} .
$$

Proof of Lemma 4.1. Without loss of generality we may assume that $i=n-1$ and $j=n$. If $x \in \mathbb{R}^{n}$ we write $x=(y, t, s)$ where $y \in \mathbb{R}^{n-2}$ and $t, s \in \mathbb{R}$. For every $y \in P_{i j}(K)$ we define the sets

$$
K_{i}(y)=\left\{s \in \mathbb{R}:(y, 0, s) \in P_{i}(K)\right\}, \quad K_{j}(y)=\left\{t \in \mathbb{R}:(y, t, 0) \in P_{j}(K)\right\}
$$

and

$$
K_{i j}(y)=\left\{(t, s) \in \mathbb{R}^{2}:(y, t, s) \in K\right\} .
$$

Then, $K_{i}(y)$ and $K_{j}(y)$ are the orthogonal projections of $K_{i j}(y)$ onto the coordinate axes of $\mathbb{R}^{2}$, and therefore

$$
\left|K_{i j}(y)\right| \leq\left|K_{i}(y)\right| \cdot\left|K_{j}(y)\right|
$$


for every $y \in P_{i j}(K)$. An application of the Cauchy-Schwarz inequality shows that

$$
\begin{aligned}
\left|P_{i}(K)\right| \cdot\left|P_{j}(K)\right| & =\left(\int_{P_{i j}(K)}\left|K_{i}(y)\right| d y\right)\left(\int_{P_{i j}(K)}\left|K_{j}(y)\right| d y\right) \\
& \geq\left(\int_{P_{i j}(K)}\left|K_{i}(y)\right|^{1 / 2}\left|K_{j}(y)\right|^{1 / 2} d y\right)^{2} \\
& \geq\left(\int_{P_{i j}(K)}\left|K_{i j}(y)\right|^{1 / 2} d y\right)^{2} .
\end{aligned}
$$

By the Brunn-Minkowski inequality, the function $\phi: P_{i j}(K) \rightarrow \mathbb{R}$ defined by $\phi(y)=$ $\left|K_{i j}(y)\right|^{1 / 2}$ is concave. Applying Lemma 4.2 with $A=P_{i j}(K), k=n-2, p=1$ and $q=2$, we get

$$
\begin{aligned}
\left(\int_{P_{i j}(K)}\left|K_{i j}(y)\right|^{1 / 2} d y\right)^{2} & \geq \frac{n}{2(n-1)}\left|P_{i j}(K)\right| \int_{P_{i j}(K)}\left|K_{i j}(y)\right| d y \\
& =\frac{n}{2(n-1)}|K| \cdot\left|P_{i j}(K)\right| .
\end{aligned}
$$

Combining the above inequalities, we get the lemma.

Remark. The estimate in Lemma 4.1 is sharp when $n=3$, as one can see by the following example: Let $K=\operatorname{conv}\left\{Q_{2}, \pm e_{3}\right\}$ where $Q_{2}=[-1,1]^{2}$ is the unit square in $\mathbb{R}^{2}$. Then, $\left|P_{1}(K)\right|=\left|P_{2}(K)\right|=2,\left|P_{12}(K)\right|=2$ and $|K|=8 / 3$.

We are now ready to prove (1.10).

Theorem 4.3. Let $K$ be a convex body in $\mathbb{R}^{n}$. For every $u \in S^{n-1}$ we have

$$
\frac{\partial(K)}{|K|} \geq \frac{n}{2(n-1)} \frac{\partial\left(P_{u^{\perp}}(K)\right)}{\left|P_{u^{\perp}}(K)\right|}
$$

Proof. From Lemma 4.1 we have

$$
\left|P_{\theta^{\perp}}(K)\right| \cdot\left|P_{u^{\perp}}(K)\right| \geq \frac{n}{2(n-1)}|K| \cdot\left|P_{\langle\theta, u\rangle}(K)\right|
$$

for every $\theta$ on the unit sphere $S\left(u^{\perp}\right)$ of $u^{\perp}$. We integrate both sides with respect to the rotationally invariant probability measure $\sigma_{u}$ on $S\left(u^{\perp}\right)$ and use Cauchy's surface area formula. The right-hand side gives

$$
\begin{aligned}
\frac{n}{2(n-1)}|K| \cdot \int_{S\left(u^{\perp}\right)}\left|P_{\langle\theta, u\rangle^{\perp}}(K)\right| \sigma_{u}(d \theta) & =\frac{n}{2(n-1)}|K| \cdot c_{n-1} \partial\left(P_{u^{\perp}}(K)\right) \\
& =\frac{n}{2(n-1)} c_{n-1}|K| \cdot \partial\left(P_{u^{\perp}}(K)\right),
\end{aligned}
$$


where $c_{n-1}=\omega_{n-2} /(n-1) \omega_{n-1}$, and from the left-hand side we get

$$
\begin{aligned}
\mid P_{u^{\perp}} & (K)\left|\int_{S\left(u^{\perp}\right)}\right| P_{\theta^{\perp}}(K) \mid \sigma_{u}(d \theta) \\
& =\left|P_{u^{\perp}}(K)\right| \cdot \frac{1}{2} \int_{S\left(u^{\perp}\right)} \int_{S^{n-1}}|\langle\theta, \xi\rangle| \sigma_{K}(d \xi) \sigma_{u}(d \theta) \\
& =\left|P_{u^{\perp}}(K)\right| \cdot \frac{1}{2} \int_{S^{n-1}} \int_{S\left(u^{\perp}\right)}|\langle\theta, \xi\rangle| \sigma_{u}(d \theta) \sigma_{K}(d \xi) \\
& =\left|P_{u^{\perp}}(K)\right| \cdot c_{n-1} \int_{S^{n-1}} \sqrt{1-\langle\xi, u\rangle^{2}} \sigma_{K}(d \xi) \\
& \leq c_{n-1}\left|P_{u^{\perp}}(K)\right| \cdot \partial(K) .
\end{aligned}
$$

It follows that

$$
\left|P_{u^{\perp}}(K)\right| \cdot \partial(K) \geq \frac{n}{2(n-1)}|K| \cdot \partial\left(P_{u^{\perp}}(K)\right)
$$

Remark. Using Lemmas 3.1 and 3.2 as in the proof of Theorem 3.3, one can obtain Lemma 4.1 and a variety of Loomis-Whitney type inequalities about "small sets" of coordinate projections of a convex body.

\section{REFERENCES}

1. H. Bergstrom, A triangle inequality for matrices, Den Elfte Skandinaviske Matematikerkongress, Trondheim, 1949. Oslo: Johan Grundt Tanums Forlag 1952. MR 14:716e

2. L. Berwald, Verallgemeinerung eines Mittelwertsatzes von J. Favard, für positive konkave Functionen, Acta Math. 79 (1947), 17-37. MR 9:13d

3. E.F. Beckenbach and R. Bellman, Inequalities, Springer-Verlag (1971). MR 33:236

4. Yu.D. Burago and V.A. Zalgaller, Geometric Inequalities, Springer Series in Soviet Mathematics, Springer-Verlag, Berlin-New York (1988). MR 89b:52020

5. A. Dembo, T.M. Cover and J.A. Thomas, Information theoretic inequalities, IEEE Trans. Information Theory 37 (1991), 1501-1518. MR 92h:94005

6. M. Fradelizi, A. Giannopoulos and M. Meyer, Some inequalities about mixed volumes, preprint.

7. Ky Fan, Some inequalities concerning positive-definite hermitian matrices, Proc. Cambridge Phil. Soc. 51 (1955), 414-421. MR 17:935c

8. L.H. Loomis and H. Whitney, An inequality related to the isoperimetric inequality, Bull. Amer. Math. Soc. 55 (1949), 961-962. MR 11:166d

9. M. Marcus and L. Lopes, Inequalities for symmetric functions and Hermitian matrices, Canad. J. Math. 8 (1956), 524-531. MR 18:877b

10. R. Schneider, Convex Bodies: The Brunn-Minkowski Theory, Encyclopedia of Mathematics and its Applications 44, Cambridge University Press, Cambridge (1993). MR 94d:52007

11. R. Schneider, On the Aleksandrov-Fenchel inequality, Discrete Geometry and Convexity (eds. J.E. Goodman, E. Lutwak, J. Malkevitch and R. Pollack), Ann. New York Acad. Sci. 440 (1985), 132-141. MR 87c:52019

Department of Mathematics, University of Crete, Iraklion, Greece

E-mail address: apostolo@math.uch.gr

Department of Mathematics, University of Crete, Iraklion, Greece

E-mail address: hmarian@math.uch.gr

Department of Mathematics, University of Crete, Iraklion, Greece

E-mail address: paouris@math.uch.gr 